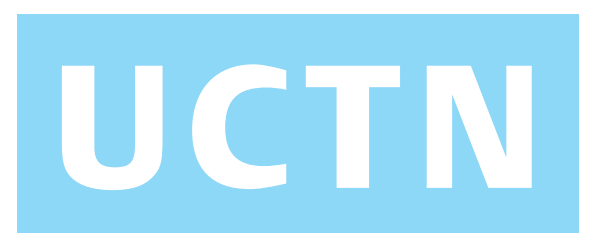

\title{
Appendicitis with a palpable mass
}

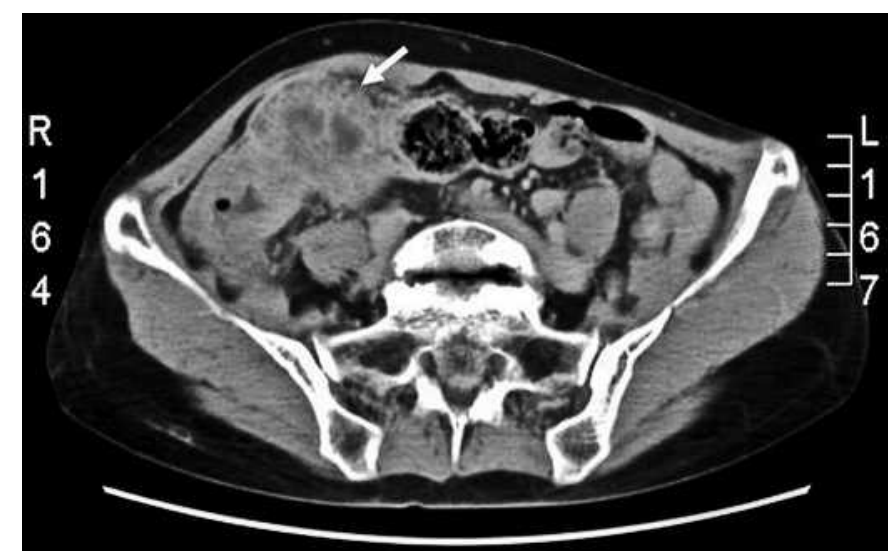

Figure 1 Contrastenhanced abdominal computed tomography, showing cecal wall thickening and an ill-defined pericecal mass lesion with marginal enhancement (arrow).
A 65-year-old woman presented with intermittent right lower quadrant pain that had persisted for more than 2 weeks. The pain was described as cramping, and was unrelated to food intake or defecation. Five days before admission, a tender mass had become palpable over the right lower quadrant. There had been no fever episodes before she came to the hospital. The physical examination was unremarkable except for a palpable, tender mass over the ileocecal area. The laboratory data were within reference ranges, except for a white blood cell count of $11700 / \mathrm{mm}^{3}$ (normal: $4500-10000 / \mathrm{mm}^{3}$ ). Tumor markers, including carcinoembryonic antigen, CA199, and CA125, were normal. Abdominal ultrasonography revealed a heterogeneous mass lesion over the right lower quadrant. Contrast-enhanced computed tomography (CT) showed cecal wall thickening and an ill-defined pericecal mass lesion with marginal enhancement (Figure 1). Because a cecal tumor could not be excluded, colonoscopy was performed, demonstrating mucosal bulging, edema, and hyperemia, with a spontaneous discharge of pus at the appendiceal orifice (Figure 2). A diagnosis of appendicitis with abscess formation was made. Conservative treatment with intravenous antibiotics was administered, and the abdominal mass was not palpable 2 weeks later. The clinical course was uneventful, and the patient remained asymptomatic during the ensuing 3month follow-up period.
Acute appendicitis is the clinical diagnosis of an inflammatory reaction in the appendix, relying on a detailed history and physical examination. However, up to one-third of patients suspected of having acute appendicitis may present with atypical clinical findings [1]. In addition, $2-6 \%$ of the patients may present with a palpable mass [2]. With a concern about possible cecal malignancy, surgeons may be faced with a dilemma when deciding on the appropriate form of management. CT and transabdominal ultrasonography have been reported to demonstrate excellent accuracy in the diagnosis of suspected appendicitis [3]; however, cecal carcinoma may present as a pericecal inflammatory mass, due to a perforation [4]. Colonoscopy therefore appears to be helpful for diagnosing appendicitis when imaging studies are nondiagnostic. With regard to the treatment of appendicitis with abscess formation, a conservative approach with routine follow-up has been recommended [5]. Appendectomy is suggested only when symptoms recur.

\section{Endoscopy_UCTN_Code_CCL_1AD_2AG Endoscopy_UCTN_Code_CCL_1AD_2AD}

\section{S. L. Yan', Y. T. Liu', D. A. Chou ${ }^{2}$}

${ }^{1}$ Division of Gastroenterology, Dept. of Internal Medicine, Show Chwan Memorial Hospital, Changhua, Taiwan 2 Division of General Surgery, Dept. of Surgery, Show Chwan Memorial Hospital, Changhua, Taiwan.

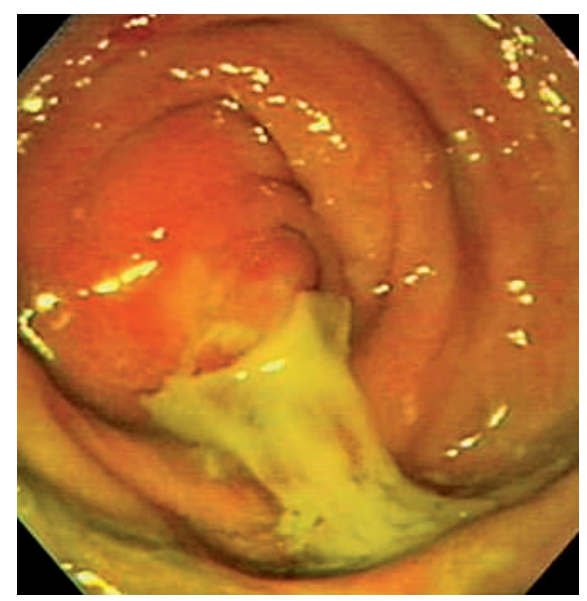

Figure 2 Colonoscopic image showing mucosal bulging, edema, and hyperemia at the appendiceal orifice, with a spontaneous discharge of pus.

\section{References}

${ }^{1}$ Berry J Jr, Malt RA. Appendicitis near its centenary. Ann Surg 1984; 200: 567-575

${ }^{2}$ Hoffmann J. Contemporary management of appendiceal mass. Br J Surg 1993; 80: 1350

${ }^{3}$ Horton MD, Counter SF, Florence MG, Hart MJ. A prospective trial of computed tomography and ultrasonography for diagnosing appendicitis in the atypical patient. Am J Surg 2000; 179: 379-381

${ }^{4}$ Poon RT, Chu KW. Inflammatory cecal masses in patients presenting with appendicitis. World J Surg 1999; 23: 713 -716

${ }^{5}$ Tingstedt B, Bexe-Lindskog E, Ekelund M, Andersson R. Management of appendiceal masses. Eur J Surg 2002; 168: 579-582

\section{Corresponding author}

\section{A. Chou, M.D.}

Division of General Surgery,

Dept. of Surgery

Show Chwan Memorial Hospital

542 Chung-Shan Road, Section 1

Changhua 500

Taiwan

Fax: $\quad+886-4-7233190$

Email: yslcsmc@hotmail.com 